\title{
Comunidad epistémica, política y filosofía del diseño en Chile: un desafío académico y profesional pendiente
}

\begin{abstract}
Resumen
Este trabajo presenta una reflexión académica sobre las dificultades que enfrenta el diseño en Chile como profesión de mercado, para constituir su comunidad disciplinar. Hace suya la necesidad de fomentar la discusión filosófica y epistemológica dentro del ámbito académico y profesional, de modo de producir sustento teórico a la actividad del diseño y otorgar legitimidad a su práctica en su capacidad de acoplamiento funcional, social y cultural. Por contraparte, se plantea la existencia de un campo fértil de posibilidades, ya que nunca hubo tanto conocimiento disponible para combinar lo local, la diversidad y lo global, e imaginar las relaciones con nuevas soluciones para expresar renovadas ambiciones disciplinarias. El trabajo se realizó a partir de un diseño de estudio no experimental, basado en observación de fenómenos en su ambiente natural. Para ello, a partir de revisión documental de las revistas de diseño existentes en Chile, los catálogos de las bienales de diseño chileno, trabajos de tesis finales de grado, conversaciones, entrevistas e investigación de campo, se plantea una investigación mixta de tipo exploratoria con alcance descriptivo de algunas características del fenómeno.
\end{abstract}

\author{
Juan Carlos \\ Rodríguez Torrent \\ Doctor en Ciencias Antropológicas \\ Profesor de la Universidad de \\ Valparaíso, Escuela de \\ Diseño-CINVIT \\ Valparaíso, Chile. \\ Correo electrónico: \\ juancarlosrodriguezt@yahoo.com \\ ㄴ) orcid.org/0000-0002-8451-2200 \\ Google Scholar
}

Rodrigo Vargas Callegari

Doctor en Sociología

Profesor de la Universidad de

Valparaíso, Escuela de Diseño Valparaíso, Chile.

Correo electrónico: rodrigo.vargas@uv.cl

๑ orcid.org/0000-0001-9076-700X

Google Scholar

Recibido: agosto 27 de 2019 Aprobado: marzo 26 de 2021

Palabras clave:

comunidad epistémica, diseño, profesión, desarrollo, ciudadanía. 


\section{Epistemic community, politics and philosophy of design in Chile: a pending academic and professional challenge}

\begin{abstract}
This work presents an academic reflection on the difficulties that Design in Chile faces as part of the professional market, to constitute its disciplinary community. It endorses the need to promote philosophical and epistemological discussion within the academic and professional field in order to produce theoretical support to the activity of design and give legitimacy to its practice in its capacity for functional, social and cultural coupling. On the other hand, the existence of a fertile field of possibilities is proposed since there has never been so much knowledge available to combine the local, the diversity and the global, and imagine relationships with new solutions to express renewed disciplinary ambitions. The work was carried out from a non-experimental study design, based on the observation of phenomena in their natural environment. For this purpose, based on a documentary review of the existing Design journals in Chile, the catalogs of the Chilean Design biennials, final degree requirement thesis, conversations, interviews and field research, a mixed exploratory research is proposed with descriptive scope of some characteristics of the phenomenon.
\end{abstract}

Key words:

epistemic community, design, profession, development, citizenship. 


\section{Introducción}

Nuestra tesis y núcleo de interés de este artículo es que frente a los problemas consensuados a nivel global, comunidades profesionales como la de diseño podrían generar mejoras sustanciales en sus reflexiones y compromisos en los territorios locales donde se desarrollan, sometiendo a revisión y examen sus prácticas. Ello requiere observar cómo se organizan y cuáles son las funciones y comportamiento esperado de los profesionales; a quiénes se debe formar, cómo y para qué problemas y mundo. Asimismo, meditar sobre la vigencia de su propuesta y valor social. Y, de modo muy substantivo, si el diseño por antonomasia es responsable y patrocinador de la vida material y artificial, no parece que pueda sustraerse a una discusión pública — desde su saber y hacer- en la procuración de planteamientos colectivos encaminados a reversar la crisis socioambiental.

Para analizar este escenario se requiere abrir una discusión en torno al caso del diseño chileno y sus dificultades para la conformación de una comunidad disciplinar, como lugar de reflexión filosófica y de generación de preguntas de interés colectivo. El objetivo de este trabajo es analizar de manera suscinta los desafíos que le imprime la sociedad actual a una profesión de mercado como diseño, en su capacidad de acoplamiento funcional, social y cultural, y su contemporaneidad como know-how, ya que su práctica dispersa no asegura sostenibilidad en el tiempo.

El trabajo se realiza a partir de un diseño de estudio no experimental, basado en observación de fenómenos en su ambiente natural, sin manipulación ni control de variables. Para ello, a partir de revisión documental de las revistas de diseño existentes en Chile $^{1}$, los catálogos de las bienales de

' Revista Base de la Universidad del Desarrollo, RchD de la Universidad de Chile, Diseña de la Pontificia Universidad Católica, y 180 de la Universidad Diego Portales. 
diseño chileno ${ }^{2}$, trabajos de tesis finales de grado, conversaciones, entrevistas e investigación de campo, se plantea una investigación mixta de tipo exploratoria con alcance descriptivo de algunas características del fenómeno.

La revisión sistemática de este conjunto de fuentes demuestra que existe muy poca evidencia acerca de una reflexión constante de modelos industriales y economía, diseño y ciclo de vida de los productos, así como de procesos de transformación de la materia (materiales, energías y residuos), la explotación de la naturaleza, o la vida urbana. Lo que nos ha permitido plantearnos el siguiente problema de investigación: ¿Cuáles son las amenazas que enfrenta el diseño chileno de no lograr consolidar una comunidad epistemológica para enfrentar los desafíos definidos local y globalmente?

En cuanto a la estructura de desarrollo de este trabajo, se intentará abordar los siguientes planteamientos: Primero, ¿cuál es el potencial de ensamblaje que actualmente posee la profesión de diseño en Chile con su ambiente? Esto obedece a que las profesiones y sustantivamente sus comunidades profesionales se caracterizaron por desarrollarse en diálogo institucionalizado con las sociedades de su época y territorio, a través de asociaciones e instituciones representativas. Segundo, pretendemos identificar el posicionamiento social de diseño devenido profesión de mercado, para intentar dilucidar la magnitud e importancia de su contribución social como entidad colectiva y simbólica. $\mathrm{Y}$, tercero, creemos importante determinar la capacidad de la comunidad de diseño para definir su propio futuro. Toda vez que fue característica fundamental de las profesiones poseer un nivel de autonomía que le permitía decidir su desarrollo, mientras las profesiones de mercado limitan su acción profesional en dar respuestas a requerimientos y solicitudes que les propone este.

${ }^{2}$ Catálogos existentes de las bienales 4, 5 y 6. De las anteriores versiones no existen catálogos oficiales, solo artículos en revistas y reportajes que también se incorporaron como fuentes. 
Discutir sobre ello, permite identificar hacia dónde se encaminan las preguntas que el diseño se formula y qué nivel de respuesta es capaz de ofrecer en Chile.

\section{Profesiones y comunidades profesionales}

Las profesiones son una de las más importantes formas de capital de nuestra época. Su desarrollo se inserta en el corazón del proyecto moderno, y comprende dimensiones económicas, sociales, culturales y políticas. Se les reconoce como modeladoras del campo intelectual, educativo y productivo de las naciones, afectando al individuo y su entorno, por lo que el estudio de sus efectos evidencia las transformaciones de una sociedad. Desde su origen, ligado al mundo eclesiástico, médico y jurídico, las profesiones se han ocupado de aumentar la calidad de vida de las personas en la sociedad, desempeñando un doble papel: el formativo del individuo y el ligado al proyecto colectivo de una sociedad civilizada (Durkheim, 2011). Por su servicio, la sociedad les retribuye en prestigio y reconocimiento por sobre la población no ilustrada.

Las profesiones se definen como "grupos expertos" que desarrollan lenguajes estructurantes y valores que tensionan y direccionan ideas, a la vez que generan hegemonías históricas que componen y forman parte de redes de relaciones, a través de las cuales se distribuye el poder mediante la práctica o habitus intelectual. Determinan relaciones de poder entre las personas (Freidson, 1986), y extensivamente hacia la naturaleza, en una reflexión de sí mismo con la exterioridad. De ahí que una lectura de estas fuerzas deba ser en relación a: 1) definir de qué se trata su proyecto y su contribución social; $y, 2)$ a través de qué preguntas aseguran su vigencia y el lugar en la agenda social, política, económica y ambiental.

Con esta consideración paradigmática entendemos la importancia que las profesiones tienen para la vida de sus agentes, comunidades disciplinares y la 
sociedad. Para mantener ciertos privilegios, están Ilamadas a cumplir con una serie de etapas de validación social: a) su delimitación permanente, ya que toda profesión debe definir qué es y cuál es su contribución social; b) la comunidad profesional debe determinar cuál es su saber exclusivo y abstracto a través del cual desarrollan sus servicios, cuestión que solo sus miembros pueden realizar; c) la reproducción de este saber específico debe hacerse por medios formales de instrucción; d) su práctica debe ser reconocida por la opinión pública como una contribución por la cual se está dispuesto a retribuir económicamente y en forma de prestigio y estatus; e) el saber monopólico debe estar en coherencia con el valor percibido de su acción; y, f) el Estado, en algunos casos, ayuda a la protección de las fronteras laborales de invasiones y usurpaciones por medio de normativas legales (Rodríguez y Guillén, 1992).

En la actualidad, producto de la creciente escolarización de la población y la alfabetización tecnológica, la obtención de un título, licencia o certificación de estudios superiores constituye un objetivo elemental para justificar competencias, por lo que la formación profesional le permite a los individuos apenas desempeñarse técnicamente en el mundo del trabajo remunerado, y hoy muchas se reconocen únicamente como profesiones de mercado (Guillén, 1990, p. 40).

En momentos donde el trabajo deja de ser físico, y cada vez se hace más dependiente de insumos y conocimientos tecnológicos (big data, robotización o inteligencia artificial), a los ojos de los profesionales de este siglo se anteponen horizontes de amenaza y precarización de las fuentes laborales de aquellos empleos basados en rutinas o de baja complejidad, dentro de un modelo de sociedad cuya regla es fagocitar de manera permanente lo que construye (Beck, 1998; Bauman, 2002). 
En otra dimensión, las profesiones deben posicionar discursos que sean relevantes socialmente, y refrendar su vigencia en los marcos de las encrucijadas locales y planetarias de tipo socioambiental. Por ejemplo, el antropoceno en la denominación de Crutzen (2002), o el mundo de la "era urbana" (UN-Habitat, 2007), corresponde a la ideología que percibe al planeta como recurso infinito; $y$, que actualmente ha alcanzado a los límites de la esfera, ya que desde la década de 1970 los costos del crecimiento han superado ampliamente a los beneficios (Latouche, 2012). Sabemos que de la mano del maquinismo, el productivismo exacerbado, la implementación de esta ideología ha llegado a alterar todos los ciclos humanos, alcanzando también a la biósfera, generando una serie de externalidades en diversas dimensiones de la experiencia socioterritorial. Y que aquello que fue el modelo de industrialización que permitió el desarrollo de Occidente y de Japón (Gorz, 1998), hoy es visto como el punto de inflexión del productivismo; lo cual deja tras de sí, las huellas de un proceso de disciplinamiento laboral, y de acceso diferenciado a las fuentes de bienestar material y social (Gorz, 1998; Garza y Salas, 2006; La Serna, 2010).

En paralelo, los sistemas tecnológicos y productivos incorporados por la modernidad occidental se han sustentado en una conceptualización que consideraba a la naturaleza como un recurso, esperando a ser explotado. Entonces, por efecto del industrialismo y las formas de consumo subyacentes, la dimensión educativa, formativa y profesional tendrá cada vez mayor impacto en la reflexión sobre los diferentes ecosistemas sociales y naturales, tal como lo indican importantes hitos, diagnósticos e investigaciones críticas como Primavera Silenciosa, el trabajo pionero de la bióloga Rachel Carson (1962); la reunión del Club de Roma ${ }^{3}$ (1968), la Conferencia de Estocolmo ${ }^{4}$ (1972) el

\footnotetext{
${ }^{3}$ Corresponde a una organización no gubernamental, a la que concurren científicos y políticos, los que previamente habían encargado al MIT un informe que diera cuenta de la relación entre crecimiento y problemas ambientales. El resultado, publicado en 1972, bajo el título The Limits to Growth, fue redactado principalmente por la biofísica Donella Meadows, y un conjunto de 17 colaboradores.

${ }^{4}$ Discute el estado del medio ambiente a nivel mundial, lo que implica un proceso creciente de conciencia política sobre la condición de la naturaleza y los ecosistemas, lo que tendría fuerte impacto en los países que componen la actual Comunidad Económica Europea.
} 
Informe Brundtland ${ }^{5}$ (1987), la Cumbre de la Tierra de Río de Janeiro ${ }^{6}$ (1992), a las que siguen una serie de iniciativas que han intentado fomentar nuevas reflexiones y desafíos.

Estas dimensiones y desafíos marcan las improntas de los campos profesionales, sus propias preguntas y compromisos para redimir este modo de producir-vivir. Parece ineludible para toda profesión contemporánea la tarea de interrogarse sobre sus mecanismos para mantenerse vigente, pero ante todo de manera sostenible. Es difícil que desde la política, la academía, el mundo profesional y la ciudadanía no exista una mirada crítica o planteamientos frente a la situación descrita, y no se asuman algunos de estos imperativos urgentes. Si bien, se subentiende que decisiones individuales dificilmente tendrán un efecto global en el corto plazo, conviene recuperar el concepto de campo propuesto por Pierre Bourdieu (1988, 2011), como el lugar en el que se confrontan las hegemonías predicativas ante el diagnóstico de los problemas, e interpelar directamente a las comunidades profesionales por preguntarse colectivamente sobre sus postulados y vigencia de los mismos, así como su capacidad para acoplarse con las necesidades y demandas de la sociedad a la que prometen dar servicio.

\title{
Antecedentes para una discusión: la profesión de diseño en Chile en la entrada del siglo XXI
}

Señala Dingwall (2004):

\begin{abstract}
Cuando tienes que movilizar capital mundialmente para construir el Canal-Túnel o el puente que va desde Suecia hasta Dinamarca, entonces necesitas ingenieros cuyas habilidades sean reconocidas por todos los banqueros internacionales y necesitarás un régimen legal y de contabilidad en el que cualquiera pueda confiar. (p. 15)
\end{abstract}

\footnotetext{
${ }^{5}$ Our Common Future, elaborado por la exprimera ministra de Noruega, Gro Harlem Brundtland.

${ }^{6}$ El principio $\mathrm{N}^{\circ} 4$ establece que, "Para alcanzar el desarrollo sostenible, la protección del medio ambiente debe ser parte del proceso de desarrollo y no puede ser considerado por separado".
} 
El elemento central es la confianza, tanto en las estructuras de la nación o en las formas globales de circulación del capital y el conocimiento. No hay forma de división social del trabajo si esta no reconoce capacidades y conocimientos comunes y portables territorialmente, y valorizados por la comunidad en un proceso de cambio y actualización permanente. Lo que habla de tres cuestiones centrales: ensamblaje, posicionamiento social y conocimientos valorizados.

Si el diseño por antonomasia es creador de la vida material y artificial, también requiere de una apreciación de esa característica profesional y confianza. No es posible, si se le considera conocimiento experto dentro de la división social del trabajo, que pueda sustraerse a coordenadas ubicadas en la vida cotidiana y como interfaz entre naturaleza y cultura, especialmente por los flujos de energía que moviliza en procesos industriales. Asimismo, a la procuración de planteamientos colectivos encaminados a reversar la crisis del modelo de desarrollo y a realizar nuevas preguntas que contribuyan a buscar soluciones y mejorar algunos de los problemas en distintas escalas.

Nuestra hipótesis de trabajo es que al diseño en Chile le ha faltado consolidación profesional como comunidad epistémica (Vargas y Rodríguez, 2019) en los tres puntos señalados. Pese a su popularidad académica ${ }^{7}$, su capacidad de respuesta es menos proactiva como para alcanzar trascendencia portable y reconocimiento, ya que está anclado en un tiempo teórico colectivo menos reflexivo del deseable, propio de un diseño mercado y de la contingencia, que descansa en la creación autoral más que en la innovación para los tiempos colectivos presentes.

\footnotetext{
${ }^{7}$ La popularidad académica refiere a que 2012 existía una matrícula de 23.042 estudiantes, casi 200 programas de estudio distintos, que impedían administrar racionalmente sus diferencias, y casi 1.000 profesores de diseño. Solo algunos de estos programas podían movilizar casi 7 millones de dólares anuales con el tamaño de la matrícula. Además, estimamos unos 60.000 egresados, lo que supera los límites de la racionalidad disciplinar (Vargas et al., 2018).
} 
La vida cotidiana y sus ciclos deben ponerse al frente. Los problemas socioambientales ingentes que ponen en riesgo la existencia misma de la vida y la noción de desarrollo han de ser revisados, actualizados y discutidos conforme al saber-hacer propio de la enseñanza, la práctica profesional y como parte substantiva de una comunidad epistémica. Se trata de cuestiones que tienen que ver con la naturaleza y las formas productivas asociadas a la construcción de la vida material, ya que la noción misma de progreso y crecimiento -ampliamente desplegada entre los años 50 y 70 del siglo pasado—- y que permitió el incremento de los niveles de bienestar material, estuvo sustentada en una importante contribución del campo a través de la artificialización de la vida.

Ahora, estamos frente a otro tipo de industrias, coordenadas axiológicas, paradigmáticas y multicriterios que definen los modos profesionales y las relaciones con la naturaleza, lo que implica replantearse el vínculo entre la interfaz de diseño y la vida individual y colectiva y los asentamientos humanos, entre la industria y la urbe, entre el trabajo y la ciudad como patrón demográfico dominante e interacción social. Mucho del replanteamiento tiene que ver con impactar en la política pública, ámbito de particular importancia para el desarrollo del campo, ya que esta refiere al conjunto de acciones, medidas, decisiones y estrategias que un Estado realiza o decide adoptar, con el claro objetivo de satisfacer un conjunto de necesidades colectivas, respondiendo a objetivos identificados como estratégicos y prioritarios (Olavarría, 2007) ${ }^{8}$.

El diseño-profesión debe dialogar con la política pública, frente a aquello que afecta negativamente el bienestar como el sobreconsumo y el mal vivir, lo que se presenta como una oportunidad observar cómo puede legitimar su saber

\footnotetext{
${ }^{8}$ La existencia de una política de Estado en diseño condiciona necesariamente el desafío de estructuración disciplinar, permeando sobre sectores industriales que aún creen que diseño participa en cuestiones menores del proceso. Baste recordar que fue Winston Churchill quien promovió el Design Council, para ayudar a recuperar al Reino Unido después de la Segunda Guerra Mundial. Su fortaleza ha trascendido, logrando posicionar al diseño por décadas en el imaginario británico.
} 
dentro de los problemas públicos como la salud del planeta y el bienestar colectivo, elaborar juicios técnicos y razonados, desarrollar propuestas inclusivas, de reconocimiento, fomento de derechos ciudadanos y también de los otros seres. Sin embargo, la confianza social (reconocimiento) en la profesión se encuentra debilitada por la ausencia de una pregunta que le otorgue identidad.

Después de que paradigmáticamente el diseño en Chile fue introducido por el Estado - a finales de los 60 y principios de los 70, como una necesidad estratégica para desarrollar la industria nacional (Bonsiepe, 1985; Medina, 2013), de la mano del modelo neoliberal ha quedado definido fuertemente por el mercado en las últimas tres décadas, y escasamente con la industria productiva. Dejó de elaborar propuestas proyectuales que comprometen paradigmas sobre las materialidades y el uso de los recursos con impacto, que ayuden a la dignificación de la vida a través de la construcción de interfaces virtuosas, identificando sujetos ejes (i.e. mujeres, hombres, niños, jóvenes) y objetos articuladores públicos (i.e. parques, plazas, barrios, espacios, juegos, veredas, mobiliario, sistemas) y privados (i.e. los subsistemas del hogar).

Se produce un enroque de escalas, desde lo colectivo a lo individual, lo que invisibiliza el peso estratégico en relación a la política pública y el bienestar. Una reorientación profesional se debe dar sobre la evaluación de la actualidad (i.e. local y regional) y sincronía profesional que posee el campo con cuestiones trascendentes (i.e. modo de producción, integración socioespacial, huella hídrica y de carbóno, servicios, valor agregado), ya que las consecuencias de un desacople de sus preguntas, de un saber sin hacer por ausencia de núcleo utópico, expresaría que los objetos resultantes son solo medios facilitadores, ya que no constituyen fines profesionales si no son ubicados en redes y constelaciones holísticas. 
En términos de Manzini (1996), el papel del proyectista y su quehacer en el desarrollo de nuevos proyectos sería a mediano y largo plazo, ubicándose en el rediseño de:

[...] todo un mundo que ya fue diseñado desde el interior de la hipótesis del crecimiento indefinido y de la irrelevancia del sustrato natural [...]. Hace falta movilizar las potencialidades de la técnica para revelar nuevas posibilidades para indicar que, en cualquier caso, el hoy necesario rediseño del ambiente artificial puede llevar nuevas calidades. (p. 108)

Según Manzini (1996), hace falta "movilizar las potencialidades de la técnica". Aquí, más bien postulamos que, en una primera etapa, es a través de la filosofía y el arte de hacer (y de refinar) las preguntas adecuadas al contexto. La confianza conferida al campo y su papel en la vida social es también en relación a fines políticos y éticos del diseño, como los contenidos en la idea del desarrollo sustentable y su tríada ecología, economía y sociedad. Si estos no se esclarecen, y no se supera lo puramente formal, quedan dos preguntas fundamentales sin responder: ¿cómo se comprende a las personas desde la perspectiva de las nuevas necesidades en un tiempo huérfano de idea de desarrollo? y ¿cómo se identifican y jerarquizan problemas y objetivos fuera de la contingencia de mercado?

Las interrogantes son básicas. Sabido es que las profesiones se desarrollan en procesos situados temporal y geográficamente, como los enunciados en la Cumbre de Río. En Italia es posible que muchos de los temas que enunciemos como problemáticos ya estén resueltos. Sin embargo, para el caso chileno, y probablemente en la región latinoamericana, es de total pertinencia la pregunta por la justificación ontológica. Si estos preceptos básicos no se aparecen con fuerza: ¿cómo se justifica, en la contingencia actual, una actividad que no es capaz de reflexionar colectivamente? De ahí nuestra hipótesis de trabajo: el diseño en Chile no ha logrado una consolidación profesional adecuada para movilizar un discurso público (Vargas y Rodríguez, 2019), debido a que 
—pese a su popularidad académica — su comunidad epistémica no constituye un referente técnico, social y político para los agentes individuales que se desempeñan profesionalmente en su nombre, ya que su relato está anclado en un tiempo teórico incompatible con los tiempos colectivos y desafíos presentes.

\section{Diseño y validación profesional}

Para que el diseño tenga estatus de profesión, en Chile debe construir una confianza básica, extramuros académicos; responder como un saber especializado y experto dentro del mundo del trabajo y de comunidades epistémicas, siempre puesto en términos de valor social. La comunidad epistémica corresponde a redes de profesionales que poseen conocimientos expertos y especializados, con competencias y experiencias en el campo, lo que les otorga legitimidad para participar en el mundo de las políticas y establecer puntos de vista, ya que poseen una episteme común que opera como guía orientadora para afianzar sus acciones. Este subsuelo de creencias permite la emisión de juicios profesionales, deliberar, influir, imponer criterios de validez y participar en la agenda política, afectando la política pública en el área de la construcción de la artificialización de la vida (i.e. sobre la calidad ambiental de los productos). En este sentido, la red como estructura compacta y limitante (quiénes y cuántos participan), que incluye otras redes en la que se reconoce la diversidad (lo que es distinto a una profesión), permite la circulación de ciertos valores compartidos, problemas y soluciones comunes a partir de procedimientos conocidos por todos. Lo que añadido al prestigio de sus integrantes, y que constituye el principal capital colectivo, es recogido por las instancias académicas y las burocracias del Estado, en cuanto las posiciones generadas por las comunidades ofrecen lecturas alternativas dentro de la agenda pública (Haas, 1992, 2004). 
La interlocución representa lo deseable, y lo que acontece en Chile en muchos campos (i.e. paradigmáticamente el Colegio Médico). Sin embargo, en el mundo del diseño predominan tres cuestiones centrales que le obstaculizan:

1. Se define, por parte del mundo académico y los estudiantes, que el diseñador es alguien que soluciona problemas (sin que aparezcan especificados, lo que podría elevar la máxima a todos).

2. Que la solución de problemas descansa en la creatividad.

3. Que lo visible y trascendente son los objetos y no las personas o la calidad de sus vidas.

Lo primero es resolver: ¿sobre qué tipo de problemas versa la acción de diseño? Lo segundo: ¿a quién preguntar en Chile?, ya que la definición de un campo creativo por excelencia, no resuelve ni explica el problema de los límites del mismo y sus impactos, ni su compromiso ético y político. Más aún, la creatividad no es patrimonio de ningún campo particular. Lo tercero: ¿trata de los objetos en sí mismos?, ¿para la vida colectiva o para cada sujeto en particular?, ¿con criterios asociados a la sustentabilidad ambiental o con fines de mercado? Por tanto, ¿qué confianza se puede depositar en una "profesión" que se piensa "singularmente" y sobre problemas de difícil anclaje?

La imposibilidad de responder estas interrogantes demuestra que no existe un rasgo profesionalizante, con un subsuelo alternativo de ideas y sin orientación definida. Y, que si las profesiones requieren ser acotadas y tener jurisdicción experta, y estar definidas por un núcleo de conocimientos exclusivos y excluyentes, este no es el caso. Todo indica, a través de la práctica docente, los resultados expuestos en las bienales de diseño y las publicaciones, que domina una modalidad de amplio espectro bajo el rótulo de diseño. Esencialmente, se reacciona a un requerimiento (en el plano de lo estético o funcional utilitario), con modelos en fase de desarrollo de concepto, desentendidos de una historia 
industrial, sin evaluación técnico-productiva, demostrando una desconexión con el medio donde se ubica el universo de soluciones de mercado a problemas complejos de la sociedad, que podrían y necesitan ser enfrentados proyectualmente.

Esto, genera tres escenarios: 1) se desenfoca respecto a dónde se encuentran los espacios para hacer contribuciones significativas en el plano profesional, limitando las posibilidades de posicionamiento frente a otras profesiones mejor arraigadas y con mayor reconocimiento por parte de la opinión pública; 2) dificulta el diálogo ínter y transdisciplinario, porque sin preguntas disciplinares no hay objetos de frontera común; 3) que el campo diseño quede alojado en el organigrama burocrático del país en el Ministerio de la Cultura y las Artes, y no en el de Economía, o en Fomento Productivo.

Sin duda, esto constituye un déficit. Solo una comunidad epistémica puede pensar y preparar proyectualmente el presente y el futuro, trazando coordenadas de confianza y credibilidad a través de objetos, sistemas y servicios que allanen la posibilidad de alcanzar bienestar material y sicológico, y profundizar un imaginario del buen vivir y la integración. Asimismo, desmaterializar y revisar las acciones productivas con multicriterios, avanzar en una perspectiva sustentada en una filosofía posmaterial, de responsabilidad ética, política y de valores trascendentes, favoreciendo el equilibrio de los sentidos, la belleza, la funcionalidad y la expedita comunicación, como ha sido propuesto por Rams como sus principios del "Buen Diseño" hace más de cinco décadas.

Identificar algunas orientaciones que ofrezcan confianza y valor, evita una perspectiva reactiva frente al mercado y al usuario del objeto. Ello introduce una nueva perspectiva bajo la denominación de un usuario devenido ciudadano, entendido como sujeto de derecho dentro de un hábitat activo y profundamente político. Un ciudadano, a diferencia de un usuario, tiene 
sentido de pertenencia, está protegido y es sujeto de derecho; es parte de una comunidad, y asume y adscribe a sus características la que le otorga un estatus y le cohesiona con los pares, y los separa de otros que no comparten esos rasgos. El usuario es el que usa un servicio, o usa ordinariamente algo sin necesariamente sentir adscripción.

El diseñador profesional puede acompañar al ciudadano en sus procesos de alfabetización y discriminación frente a las materialidades que usa y manipula. Por lo que conscientes de algunas dificultades producidas por la sociedad industrial y posindustrial, el diseño chileno en su tránsito sobre el presente debe asumir que es imperativo organizarse de acuerdo a un proyecto alternativo en términos productivos, valóricos y de convivencia, ya que el modelo del desarrollo actual está para muchos indefectiblemente agotado. Entonces, como comunidad epistémica y profesional se puede postular como fin social a una vida bajo otras coordenadas, desde donde explorar y proponer otros modelos como el "decrecimiento" (Taibo, 2014), el desarrollo endógeno y la economía circular y de kilómetro cero, en los marcos de las potencialidades regionales y locales. Y, proponer políticas públicas hacia una intervención eficiente y eficaz sobre los problemas identificados en la interfaz, mostrando sensibilidad, promocionando una familiaridad con el artefacto y eliminando la condición pasiva de "consumidores".

\section{Dispersión del diseño, desafíos y posibilidades en el medio urbano}

La ciudad es sin duda la producción humana más importante, consistente y exitosa como ha planteado Robert Park (1999), ya que en ella se plasmó la posibilidad de rehacer el mundo en el que se vive. Es también un lugar que oprime, aliena, separa y excluye. El reto de la comunidad epistémica es cómo contribuir a reorganizar la mirada frente a la complejidad de los mundos cotidianos, con actos proyectuales reorientados hacia la pluralidad, 
la diversidad, la integración y las diferencias de condiciones de vida que esconden, y observar el impacto de cada decisión en la construcción del habitar en su sentido político. Corregir con juicios técnicos y razonados aquello mal evaluado, impropio, violento, discriminador, negador de principios de solidaridad, es lo que avala también el derecho a cambiarlo9? .

Como lo propuso el movimiento moderno en su momento, habitar y vivir imponen como reto opciones y estrategias innovadoras que articulen sustentabilidad y calidad de vida. Las ciudades chilenas están marcadas por la segregación espacial y económica, el envejecimiento y la degradación. La especulación y el extractivismo urbano han afectado la disposición del espacio público como lugar de encuentro, que permita dar contenido virtuoso al tiempo y el derecho a la ciudad; construir interacciones y tejido social para que la gente piense y se apropie del espacio a través de tiempos familiares y de ocio, es un desafío. De modo evidente, la realidad vivida pasa a constituirse en realidad social y políticamente construida, convirtiéndose en espejo de las sociedades que las producen, es decir, por omisión, con mal diseño. El espacio se vive de forma diferenciada a solo unos metros de distancia, constituyendo abismos sociohistóricos de díficil conciliación. Porque en las democracias emergentes del mundo contemporáneo no todos los ciudadanos gozan de iguales posibilidades para ejercer sus derechos. Las poblaciones subalternas son solo "tenuemente, e incluso, ambigua y contextualmente, portadores de derechos ciudadanos en el sentido imaginado por la constitución" (Chatterjee, 2011, p. 216).

\footnotetext{
${ }^{9}$ Desde 2007 más de la mitad de la población mundial vive en ciudades, y en dos o tres décadas llegará al 75\%. Lo que presiona la sostenibilidad de cualquier sistema político, económico y de convivencia social. En Chile, de acuerdo con el último Censo de Población (2017) del Instituto Nacional de Estadísticas de Chile (INE), en un universo de 17'574.003 habs., la población urbana es de un 87,8\%, frente a 12,2\% de población rural (www.censo2017.cl/descargas/home/sintesis-de-resultados-censo2017.pdf).
} 
Nos parece, las urbes _llamadas también postciudad, ciudad difusa, metápolis, ciudad global, ciudad informacional, ciudad región (véase Carrión 2016, p. 34) — deben ser leídas por una comunidad epistémica de diseño como objetos, servicios y sistemas de diseño. Sin embargo, se aprecia la ausencia de peritajes profesionales que constituyan ángulos de interlocución legitimados, capaces de revelar las dificultades en infraestructura, equipamiento y servicios; los problemas en subsistemas básicos como transporte y espacio público, distribución y tratamiento de aguas, recolección de residuos domiciliarios, energía y viviendas apropiadas. Los problemas están inventariados y demandan pensamiento estratégico y proyectual que opere articuladamente sobre aquello que materializa las definiciones en el espacio público y el derecho a la ciudad, pero que también se recojan como un marco para generar sistemas de oportunidades para el posicionamiento profesional del diseño.

El déficit de las ciudades chilenas es también el déficit de un diseño que no llega hasta ellas; una falla como profesión de su comunidad epistémica. La urbe, como laboratorio, se ofrece como un lugar para desarrollar comunidad epistémica, ya que en la metodología proyectual están los insumos de la planeación de lo artificial y de las escalas de la interacción humana. El "hecho urbano", es decir, lo que está al frente del diseño para ser configurado como concepto, "radica precisamente en los modelos de crecimiento, de renovación urbana, de rehabilitación y de regeneración basados en nuevas cualificaciones y clasificaciones que transfieren partes esenciales de los beneficios a la definición de los nuevos circuitos de la ciudad del futuro" (Nadal i Farreras, 2010, p. 182).

Otorgar lugar a la comunidad epistémica en estas reflexiones se vuelve relevante para la sostenibilidad misma del campo, repensando los modos actuales del comercio, la industria y la distribución de servicios. Ayudaría a mejorar los modelos proyectuales en torno al proyecto del buen vivir, la seguridad, 
el bienestar, el respeto al medio ambiente y las relaciones equitativas de producción. Reconceptualizar colectivamente el diseñar lo artificial en, sobre y para la ciudad, debe fundamentar — en buena parte — la ciudad que queremos: participativa, inclusiva, segura, diversa, sostenible, productiva, educadora y habitable. Entonces, para no quedar atrapado en un proyecto disciplinar anacrónico, o subsumido al puro mercado, una opción proyectual proactiva debe avanzar en la gestión de la vida como núcleo utópico, reduciendo la simplificación sectorial de una política productivista, lineal, fragmentaria y de oferta y demanda.

De hecho, Chile se autoimpuso 303 metas para lograr el objetivo de ser carbono neutral al año 2050 y resiliente al cambio climático, priorizando áreas como energía, transporte, construcción, minería, silvoagropecuaria, residuos y economía circular, infraestructura, bidiversidad, recursos hídricos, borde costero, océano, entre otras.

\section{Una propuesta: el diseño profesión en busca de nuevos autores}

En Chile, la acción profesional de diseño no puede estar separada de los asuntos políticos, del tipo de lazos que se quieren construir, los valores asociados a la convivencia humana y el resto de los seres, los estilos de vida, las cuestiones estéticas y las de orden tecnológico. El campo pierde prestancia profesional sin preguntas y con bienes superfluos, banales y efímeros, por lo que requiere plantearse en diálogo con nuevas industrias de la innovación, y orientarse hacia fuentes laborales reales, que se concentran en esfuerzos estratégicos. La actualización de sus interrogaciones y objetivos debe darse dentro de principios de integración con una ciudadanía participativa, como a través de la co-creación; ya que es una condición asociada al morar, habitar entre y con los objetos, los sistemas y los servicios como complemento. 
Proyectar la producción, distribución y consumo de bienes y servicios de manera equitativa corresponde a un imperativo ético y político para el diseño chileno, pero también para su sobrevivencia. Desarrollar interfases para que desde la construcción de la artificialidad acontezca, y generar el conocimiento, los métodos y el manejo de tecnologías ad hoc para que ello ocurra, es obligación de la comunidad epistémica en tanto agente de cambio.

Algunas cuestiones a considerar desde un nuevo diseño profesión y dentro de una comunidad epistémica, están asociadas al menos a cuatro dimensiones:

1. Comprender, participar y dialogar con un proyecto global de desarrollo, que responda a las necesidades de nuestros entornos cotidianos y ciudades pauperizadas, para potenciar el rol de ciudadano y no de usuario, acogiendo y dando respuesta a nuevas demandas.

2. En los procesos académicos, formativos y profesionales integrar vectores clásicos de su saber y práctica, pero también los contemporáneos y de interdisciplina sobre la lógica de la comunidad epistémica y no de impulsos individuales y de mercado.

3. Hacer frente propositivamente a la sociedad de la información, esa que está a la mano de todos, que expresa vigorosamente imágenes, comunicaciones y transporte que redefinen memorias, tiempos y lugares.

4. Dentro del espacio urbano, conjugar el sentido profesional en sus iniciativas proyectuales en términos de: a) memoria como reservorio del sentido de la comunidad; b) acuerdos, en el sentido de precipitar opciones para asegurar reconocimiento, tolerancia, derechos y responsabilidades; $y$, c) proyecto, entendido como imaginario de futuro sobre el cual se trabaja colectivamente por la ciudadanía.

Claramente, las interacciones en los espacios definen situaciones, las crean y las estimulan; o, en su ausencia, las anulan y minimizan. El diseño debe 
pensar intencionadamente la vida en las ciudades y la circulación, ya que debe perfilarse como un lenguaje necesario de y para la urbe. Desarrollar una hermenéutica del sentido, en tanto búsqueda de seguridad, integración, reconocimiento, empleo y bienestar, refundaría su lugar, y lo ubicaría en un lugar político y de enunciación sobre escalas de influencia profesional en la micrósfera, mesósfera o macrósfera (Maldonado, 1999).

Lo anterior, hace evidente que las ciudades exitosas se diseñan. Se construyen en escalas de proximidad física y semántica con los individuos, concentrando los centros de producción, distribución y circulación de productos, ya que están las infraestructuras, servicios especializados, de generación de conocimiento y personal calificado. En ellas hay una filosofía circulante, donde la ciencia, la tecnología y la innovación son esenciales para construir la dimensión reflexiva de lo artificial. El proyecto de diseño puede presentarse como la síntesis entre el saber y el hacer alojado en diferentes campos en la producción de impactos positivos y mensurables en y para el buen vivir. Un pensamiento proyectual urbano será complejo como la ciudad en la actualidad, por oposición al "paradigma simplificador" (Morin, 2001), a la "inteligencia ciega" que evade la complejidad de la sociedad contemporánea; "el error, la ignorancia, la ceguera, progresan, por todas partes, al mismo tiempo que nuestros conocimientos" (Morin, 2001, p. 27). Agrega Morin: "La causa profunda del error no está en el error de hecho (falsa percepción), ni en el error lógico (incoherencia), sino en el modo de organización de nuestro saber en sistemas de ideas (teorías e ideologías)" (p. 27).

No se trata de disipar la complejidad de los fenómenos y transformarlos en un orden simple, sino hacerse cargo de la confusión, la incertidumbre y el desorden (Morin, 2001). El acto proyectual en diseño, entonces, es un dispositivo para producir filosóficamente la contención del tiempo; trata de lo planeado para producir efectos materiales en coherencia con la vida en 
común, otorgando soporte a la relación de sujeto a sujeto, proyectando para otro, que considera la existencia de otro que recibe y experimenta lo que un otro concibió. Diseñar de y para la ciudad y la vida cotidiana es ideación y planeación de lo político y de una forma de gobernanza. Fundamenta una provocación que conduce a la proyectación al goce y al derecho ciudadano a tener una experiencia del tiempo, permitiendo la transformación del Homo laborans al Homo ludens — señala Hall (2003, p. 10)—.

El diseñador, en tanto se reconoce en una comunidad epistémica, ayuda a dimensionar las escalas y proximidades entre ciudadanos frente a un territorio, para generar ambientes y paisajes positivos. Volviendo "nuestra consciencia hacia el mundo y hacia nuestro propio sentido del yo y del ser [...] [permitiendo que] tengamos una experiencia del nosotros mismos como seres corporales y espirituales. De hecho, ésta es la gran función de todo arte significativo" (Pallasmaa, 2006, p. 11).

La interfaz debe expresar la necesidad de las dimensiones de las veredas para invitar al caminar pausado, la frecuencia de las flores y sus colores, dónde se deben plantar los árboles, la distribución de las sombras, el adecuado número y distribución de estacionamientos para bicicletas, la disposición de descansos en el espacio público, la proporción de comercios deseables en un barrio, las extensiones de la mesa para regular la intimidad de una conversación, los materiales y texturas que permiten administrar el tiempo del confort; aquello que cuando ideó la taza del café, con su brillo o su pátina, no solo pensó en contener el líquido, sino que proyectó la navegación de ideas entre personas que buscan una forma para fragmentar el tiempo. Lo vinculante está asociado a la gestión frente a lo que está definido por el movimiento y la inestabilidad, lo que constituye el material de la reflexión urgente. Diseñar como comunidad epistémica es pensar, imaginar, proyectar y dibujar horizontes utópicos; inexistentes y nuevos para el buen vivir, para hacer visible lo social. 
Cuando el acto proyectual ordena los factores para la producción, también opera sobre la conducta de los ciudadanos. Carga con exigencias vitales y morales; instala una experiencia espacial del tiempo, que ofrece diversas sensaciones sobre la luz, el sonido y el ritmo. Como reflejo material de la vida, el diseño representa el orden social, el poder y la cultura, y está llamado a proyectar confianza y enriquecer la condición humana y el mundo a través de la propuesta del uso organizado de los objetos en cuestiones funcionales, formales, tecnológicas, ambientales y sociales. Se concentra en la habitabilidad, la vida cotidiana, la distribución del tiempo, la seguridad, la vida social; se hace cargo de las "distintas permanencias" y las "diferentes estabilidades" que concentran la experiencia y la atención de cada uno en el fluir de la ciudad.

\section{Conclusiones}

Nuestras preguntas iniciales apuntaban a identificar qué desafíos se imponen al campo, la claridad respecto de su proyecto profesional, y su vigencia y lugar en la agenda social.

Sin temor a equivocarse: la versión chilena del diseño encuentra actualmente en la industria de la educación superior su lugar más recurrido. Sin embargo, con los límites que le impone hacia la industria productiva y la economía, lo que produce disminuición en su capacidad de interlocución inter y transdisciplinar, dificulta la constitución de una comunidad epistémica y la proyección de confianza hacia la opinión pública sobre su saber-hacer.

Para una condición distinta, el campo profesional requiere replantearse sus preguntas y su lugar dentro de los problemas de la crisis del desarrollo y la era urbana, ya que las ciudades son la caja de resonancia donde la reflexión filosófica como arte de construcción de sentidos y apertura a nuevos planos, coordenadas y dimensiones, alcanza sentido práctico. La profesión de diseño, 
dentro del sistema social nacional, requiere para cambiar su estatus del fortalecimiento de una comunidad epistémica que combine armónicamente creación e innovación, cuestión fundamental para la adquisición de una subjetividad simbólica para sus profesantes, desde donde encarar el proyecto profesional.

Resulta difícil encontrar semánticamente la crítica autorizada, los acuerdos y el control sobre las referencias circulantes que permitan responder: ¿cuándo en Chile estamos frente a un buen o mal diseño?, ¿cuáles debieran ser las convenciones sobre sus impactos esperados en la cotidianeidad, la ciudad, el espacio público y la naturaleza? Asimismo, ¿a quién y qué le quieren hablar los diseñadores y diseñadoras?

La pregunta política por excelencia es el lugar que ocupan las personas en las decisiones profesionales. La aplicación de cualquier política de desarrollo de infraestructuras, sistemas y servicios para la ciudad, no es solo un orden tecnocientífico. Ante todo, se trata de cómo se producen también órdenes sociales a partir de las decisiones, y cómo se ayuda a la construcción de ciudadanía, ya que se está modificando el espacio desde donde se construye el proyecto de vida.

Diseño visto como una metodología, o un atributo añadido final, puede ser absorbido por campos epistémicos más consolidados. Si no es capaz de identificar un núcleo utópico, de construir sus fronteras de jurisdicción y no incrementa substantivamente su espesor epistemológico, pone en riesgo la supervivencia del campo al no ser reconocido por sus públicos y las industrias y servicios que demandan diseño. Para el campo disciplinario es importante la reflexión filosófica, a fin de movilizar el lenguaje de lectura que refrende los usos, compromisos y objetivos del saber-hacer; interrogarse y responderse permanentemente acerca de la funcionalidad del diseño como profesión, su 
responsabilidad social y esclarecer bajo qué contenidos y narrativas se trata de una disciplina que se orienta efectivamente al bien social; o, cuándo se trataría de una radical impostura, una acción eminentemente especulativa o puramente "creativa" e "individual", ligada a oportunidades de mercado.

Una madurez profesional para diseño chileno, es decir, de una comunidad epistémica que señale claramente su especificidad y transmita confianza, solo podría alcanzarse: a) si es capaz de definir un área de competencia exclusiva, un cuerpo de preguntas vigentes y en tono con problemas estructurales, y modos consensuados de responder al proyecto de bienestar colectivo; b) si desarrolla un pensamiento filosófico propio para establecer fundamentadamente sus compromisos capitales frente a la sociedad, explicitando modos profesionales comunitarios en diálogo con otros campos que desarrollen preguntas similares; y, c) pueda convocar a una comunidad epistémica que regule los límites profesionales, que desarrolle mecanismos de control de la reproducción; o, finalmente, defendamos que se trata de una ocupación de posindustria no sometida a los cánones tradicionales de una profesión.

\section{Referencias}

Bauman, Z. (2002). Modernidad líquida. FCE.

Beck, U. (1998). La sociedad del riesgo. Hacia una nueva modernidad. Paidós.

Bonsiepe, G. (1985). El Diseño en la periferia. Gustavo Gili.

Bourdieu, P. (1988). Cosas Dichas. Gedisa.

Bourdieu, P. (2011). Las estrategias de la reproducción cultural. Siglo XXI.

Carrión, F. (2016). El reto político de la ciudad Latinoamericana. En L. Álvarez, G. Delgado y A. Leal (Coords.), Los desafíos de la ciudad del siglo XXI (pp. 25-41). Senado de la República-UNAM-CIICH-PUEC-PUES. 
Carson, R. (1962). Silent spring. Houghton Mifflin - The Riverside Press.

Chatterjee, P. (2011). La política de los gobernados. Revista Colombiana de Antropología, 47(2), 199-231.

Crutzen, P. (2002). Geology of mankind. Nature, 475.

Dingwall, R. (2004). Las profesiones y el orden social en una sociedad global. Revista Electrónica de Investigación Educativa, 6(1). http://redie.uabc.mx/vol6no1/contenido-dingwall.html

Durkheim, E. (2011). La división del trabajo social. Ediciones Libertador.

Freidson, E. (1986). Professional Powers. The University Chicago Press.

Garza, E. de la y Salas, C. (Coords.). (2006). La situación del trabajo en México, 2006. Universidad Autónoma Metropolitana, Rectoría General, Instituto de Estudios del Trabajo, Centro Americano para la Solidaridad Sindical Internacional, AFL-CIO, Plaza y Valdés.

Gorz, A. (1998). Miserias del presente, riqueza de lo posible. Paidós Ibérica.Haas, P. M. (1992). Introduction: Epistemic communities and international policy coordination. En P. M. Haas (Ed.), Knowledge, Power; and International Policy Coordination (pp. 1-37). University of South Carolina Press.

Guillén, M. (1989) Profesionales y burocracia: desprofesionalización y poder profesional en las organizaciones complejas. Revista Española de Investigaciones Sociológicas, 51/90, p. $35-51$.

Haas, P. M. (2004). Science policy for multilateral environmental governance. En N. Kanie y P. M Haas (Eds.), Emerging forces in environmental governance (pp. 1 15-136). United Nations University Press.

Hall, E. (2003). La dimensión oculta. Siglo XXI.

La Serna, C. (2010). La transformación del mundo del trabajo: representaciones, prácticas e identidades. CLACSO.

Latouche, S. (2012). Lâge des limites. Fayard.

Maldonado, T. (1999). Hacia una racionalidad ecológica. Ediciones Infinito.

Manzini, E. (1996). Artefactos: Hacia una nueva ecología del ambiente artificial. Celeste Ediciones. 
Medina, E. (2013). Revolucionarios cibernéticos. Tecnología y política en el Chile de Salvador Allende. LOM.

Morin, E. (2001). Introducción al pensamiento complejo. Gedisa Editorial.

Nadal i Ferreras, J. (2010). Ciudad acabada, ciudad renovada. En F. Manito (Ed.), Ciudades creativas. Volumen 2. Creatividad, innovación, cultura y agenda local (pp. 173-182). Fundación Kreanta, España.

Olavarría, M. (2007). Conceptos Básicos en el análisis de las Políticas Públicas. Documentos de trabajo Nº11, INAP-Departamento de Asuntos Públicos, Universidad de Chile, Santiago.

Pallasmaa, J. (2006). Los ojos de la piel. La arquitectura y los sentidos. Gustavo Gilli.

Park, R. (1999). La ciudad y otros ensayos de ecología urbana. Del Cerval.

Rodríguez, J. y Guillén, M. (1992). Organizaciones y profesiones en la sociedad contemporánea. REIS, 59, 9-18.

Taibo, C. (2014). ¿Por qué el decrecimiento? Un ensayo sobre la antesala del colapso. Los libros del Lince.

United Nations Human Settlement Programme (UN-Habitat). (2007). The state of the world's cities Report 2006/2007-30 years of shaping the Habitat agenda. London: Earthscan for UN-Habitat.

Vargas, R. y Rodríguez, J. (2019). Profesionalización del Diseño en Chile, una sinfonía en cuatro movimientos. RChD: creación y pensamiento, 4(6), 1-13.

Vargas, R., Rodríguez, J. y Arias, P. (2018). Diseño, sobrediseño y comunidad epistémica. Una discusión sobre los límites del campo. Base, 3, 268-277.

Cómo citar: Rodríguez Torrent, J. C. y Vargas Callegari, R. (2021). Comunidad epistémica, política y filosofía del diseño en Chile: un desafío académico y profesional pendiente. Revista KEPES, 18(24), 79-105. https://doi.org/10.17151/kepes.2021.18.24.4 